\title{
The Nature and Multiscale Techniques for Characterization of Mechanical Properties: from Nanostructured Materials to Single Macromolecules
}

\author{
Part II. Strength of Low-Dimensional Materials
}

(Continued) ${ }^{*}$

\author{
Yu.I. Golovin
}

\author{
Institute for Nanotechnologies and Nanomaterials, G.R. Derzhavin Tambov State University, \\ 33, Internatsionalnaya St., Tambov, 392000, Russian Federation; \\ Faculty of Chemistry, M.V. Lomonosov Moscow State University, \\ 1-3 Leninskiye Gory, Moscow, 119991, Russian Federation
}

Tel.: +7 (4752) 5326 8; +7 (495) 939-16-82;E-mail: golovin@tsu.tmb.ru; lunin@direction.chem.msu.ru

\begin{abstract}
The paper analyzes the experimental data on the mechanical properties of low-dimensional objects and materials: film coatings, thin near-surface layers, superlattices, nanopillars, fibers, nanotubes and macromolecules. The dependencies of the hardness, yield stress and strength on the grain size are considered for nanocrystalline materials. The range of applicability of the Hall-Petch ratio and physical factors causing deviations from it are discussed. The dynamics of the behaviour of single molecules under the action of increasing or constant applied force is considered. Estimates of the role of the mechanical properties of biological macromolecules in the functioning of living systems are presented.
\end{abstract}

\section{Keywords}

Carbon nanotubes; filaments; macromolecules; mechanical properties of low-dimensional materials; pillars; superlattices; thin films.

(C) Yu.I. Golovin, 2016

\section{Experimental results}

As specific examples of the results obtained in recent years, let us consider the most interesting, in our opinion, experimental data, in terms of simple classification of objects and tasks on the basis of their dimensions: bulk three-dimensional (3D), thin-film or two-dimensional (2D), rod-like or one-dimensional (1D) and point or zero-dimensional (0D) nanomaterials.

\section{Bulk nanostructured materials}

The first information about synthetic bulk nanomaterials appeared about half a century ago. First P. Duwes and coworkers from Caltech (USA) obtained the discs of amorphized alloys, while throwing the melt droplets on the highly thermo-conducting substrate $[99,100]$. Then A. Inoue, T. Masumoto and his colleagues offered a more convenient continuous technique of melt spinning - fast $\left(\sim 10^{6} \mathrm{~K} / \mathrm{s}\right)$ cooling of the melt stream on the surface of a rotating metal drum [99-102]. Later, many other ways of amorphization of metals and alloys were suggested [100-104], but

\footnotetext{
${ }^{*}$ Part 1. No. 1, 2016.
}

solidification of the melt remains one of the most popular techniques that is suitable for producing metallic glasses of different composition [99-111]. In the early studies that used melt spinning technique, tapes with a thickness of no more than 20-30 micrometers were obtained. However, since the mid-1990s, there have been synthesized several types of multicomponent alloys based on $\mathrm{Zr}, \mathrm{Ti}, \mathrm{Mg}, \mathrm{Ca}, \mathrm{Fe}$, $\mathrm{Co}, \mathrm{Ni}, \mathrm{Cu}$, lanthanides and noble metals which do not require such high cooling rates and allow to obtain ingots with a centimeter cross section $[100,103,112$ 114]. (Amorphous alloys are reasonably referred to nanostructured materials on the basis that they usually have in their structure ordered atomic clusters with sizes of about $1 \mathrm{~nm}$, immersed in an amorphous matrix $[99,101])$. In a number of studies [99, 115-120] the trend, called the controlled crystallization of amorphous alloys is developed. It allows in a controlled manner to grow in the amorphous matrix stochastically scattered crystal nanograins with a size from one to tens of nanometers, which lower the fragility of glass without significantly reducing the strength (only within the limits determined by Weibull statistical relationship [121]). 


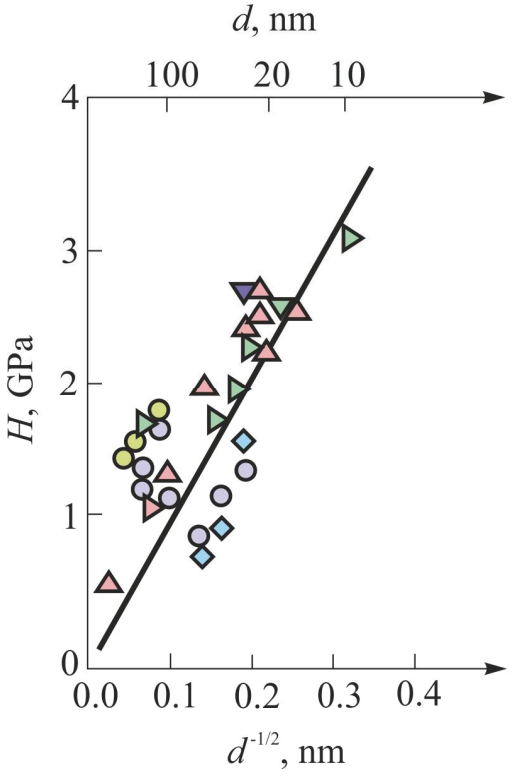

a)

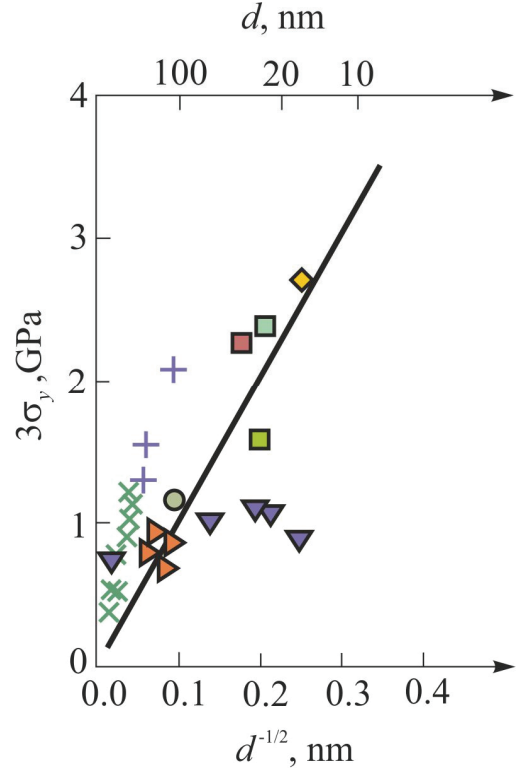

b)

Fig. 12. The dependence of hardness $(a)$ and estimated yield strength $(b)$ on the grain size for $\mathrm{Cu}$ in Hall-Petch coordinates according to different authors (different signs) [28]

In the early $1980 \mathrm{~s}, \mathrm{H}$. Gleiter proposed and then developed a method for producing nanocrystalline materials by evaporation of raw materials, vapor condensation on the cooled drum and further vacuum compression of the resulted nanopowder into the consolidated bulk nanostructured material [122]. Numerous followers continue to improve this approach $[16,35,37]$. Similar technology is widely used for synthesizing ceramic products by sintering nanopowders either pre-micronized or obtained by solgel method $[33,38]$. Great progress has been made in improving and putting in practice nanocrystalline metals and alloys, obtained by the methods of megaplastic (severe) deformation up to the value $\varepsilon=5-10$ [12]. Finally, in the last 1.5-2 decades, a large number of methods of synthesizing composite nanomaterials with a polymeric, ceramic and metal matrix have been developed [13, 33, 34].

All of the above classes of materials are characterized by the presence of nanoscale 0D morphological units (nanocrystals, nanophases, ordered units of the macromolecules in polymers) and a large number of boundaries between them per unit volume. The grain and interphase boundaries have a finite thickness (in nanostructured inorganic materials it is usually about $1 \mathrm{~nm}$ ) and the properties that are very different from the grains themselves (though the grains themselves, being nanoscale, differ significantly in structure and properties from macroscopic analogues). Grinding the structure results in the increase in the share of the material located in the boundaries and triple junctions. All this taken together leads to a strong dependence of mechanical, transport, magnetic, optical, and other properties of these materials on the size of grains, phases, and domains and structures of the grain boundaries.

This section of nanophase materials science is extensively presented in review articles and monographs $[16,21,26,29,35,37$, $39,47]$. Let us add to this list some of the most interesting, in our opinion, publications that came out in the last couple of years [123-129], and briefly sum up the results.

1. Reducing the grain size $d$ in polycrystalline single-phase materials (or particles of the second phase in multiphase materials) from tens of micrometers (which is typical of traditional materials) to several hundreds of nanometers in nanocrystalline materials causes increase in most strength characteristics, in particular $\sigma_{y}, \sigma_{b}, H$ (Fig. 12) in accordance with the empirical Hall-Petch relationship (7). This leads to multiple hardening of the material (for soft metals more than ten-fold). There have been proposed a number of models describing elementary processes with dislocations and grain boundaries, with the experiment in the above range $d$ $[16,27,30,35,37,39]$. Note that based only on the fact of consistency of experimental data with HallPetch relationship, you can not select and verify a particular theory of SE, as many of them predict the same dependence close to (7), although they proceed from completely different physical assumptions.

2. In the range of grain size $d \lesssim 20 \mathrm{~nm}$, as a rule, there are significant deviations of experimental dependences from Hall-Petch relationship, and at size $d \lesssim 10-20 \mathrm{~nm}$ there occurs their saturation or even softening with a decrease in $d$ (Fig. 13). Formally, such a variation in $\sigma_{y}(d)$ or $H(d)$ can be explained by a decrease in $k$ in equation (7) up to zero and even negative values (Fig. 14), as many authors reasonably do [47]. Other, no less authoritative experts [16] believe that in the range of small $d$ it is not $k$ that changes but the exponent $n$ (up to the sign change).

At $d \lesssim 30 \mathrm{~nm}$ the Young's modulus $E$ can start decreasing, which is due to the growing share of the material located in the grain boundaries and having less rigidity than the grains themselves. 
According to the authors [16, 27], anomalies in the Hall-Petch dependencies are caused by the fact that with decreasing $d$ ever greater role is played by accommodative contact processes and deformation in the near-border regions. It should be taken into account when introducing the additional member with $n=-1$ into Hall-Petch relation, which decreases the slope at small $d$ :

$$
\sigma_{\mathrm{c}}=\sigma_{0}+k d^{-1 / 2}-k^{\prime} d^{-1} \text {. }
$$

There are other more complicated methods for correcting Hall-Petch relationship for small $d$, taking into account the real hierarchical structures and mechanisms of plastic deformation at each structural level and leading to an increase in the number of members in relationships of type (8). Anyway, the simplest expression (7) ceases to describe experimental data at $d \lesssim 10-20 \mathrm{~nm}$, and, apparently, it makes no sense to refer to and associate the modified expressions for $\sigma_{\mathrm{c}}(d)$ with Hall-Petch relations modifying them up to complete opposites. It is more constructive to identify the causes of SE (abnormal in terms of the theories that predict relationships of HallPetch type) and appeal to other alternative mechanisms of plastic deformation, which are beginning to prevail in the nanoscale. It is reasonable to believe that region $d$ is dominated by nondislocation modes of plasticity, controlled by slip and rotation of grains, diffusion of point defects at grain boundaries and others $[3,16]$. In recent years complex models that reflect the complexity of a number of self-consistent processes ensuring the plasticity of nanocrystalline materials have become increasingly popular. Finally, in region $d$ of less than several nanometers plastic deformation becomes possible, apparently, only due to the movement of excessive point defects and their smallatomic clusters [52].

In most practical applications it is necessary to provide a combination of high strength and fracture viscosity. For this purpose, a special design of grains with optimal angles of disorientation, type of boundaries and their structure is developed. The grains are reinforced with nanoparticles of another phase or transformationally reinforced inclusions (such as in ceramics on the basis of zirconium dioxide [130, 131]) are created. Besides nanotwins can be introduced into nanograin bodies $[27,28,132,133]$ to achieve bimodal character of size distribution of the grains [134, 135], etc. In the area of composites special measures are made to increase the adhesion of single phases, reduce porosity, internal and thermal stresses, dissolution and chemical degradation of contact nanophases.

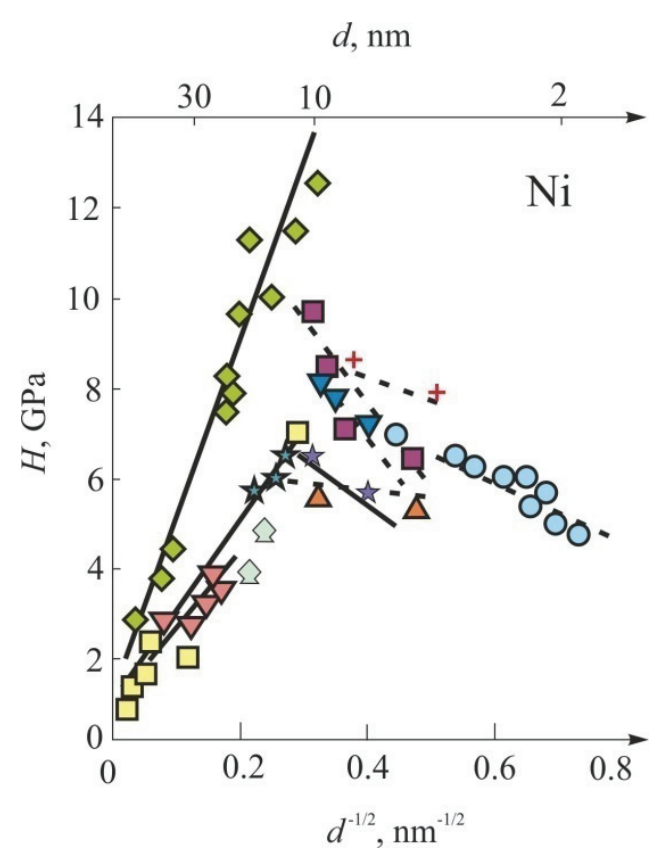

Fig. 13. The effect of a grain size on the nickel hardness in the size range between $2 \mathrm{~nm}$ and above [3]

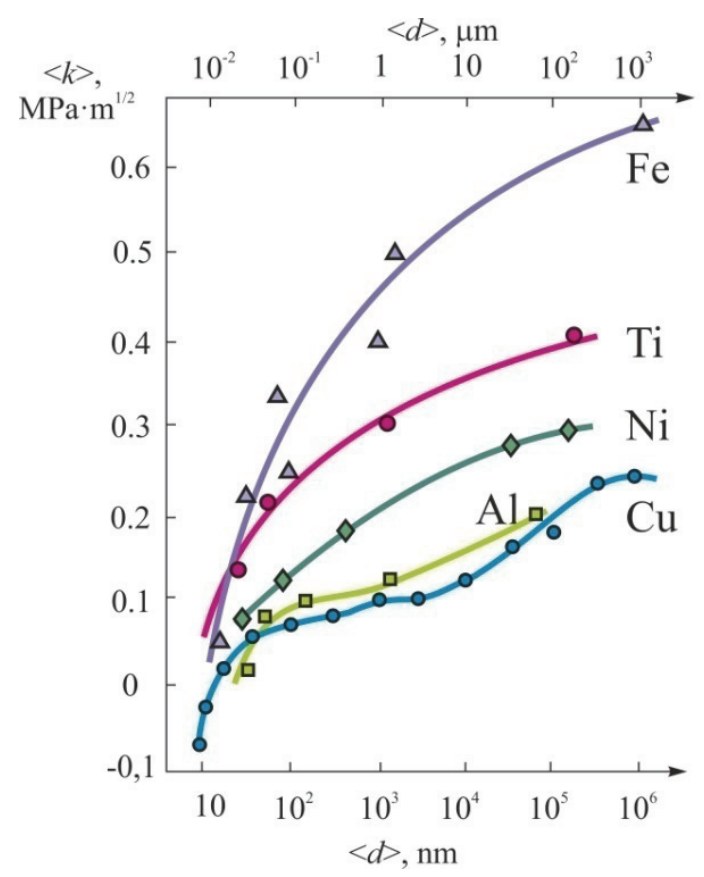

Fig. 14. The dependence of the average value of the Hall-Petch coefficient $<k>$ on the average grain size $<_{d}>$

\section{Thin films and coatings}

A large variety of methods for producing thin coatings (usually classified into two groups - PVD and CVD, which mean physical or chemical deposition techniques, respectively) allows varying their composition and properties in much wider ranges than those of bulk nanostructured materials [136, 137]. Similar to bulk materials, thin films are influenced by dimensional effects of different nature. For tribological 
coatings, in addition to the known causes of the yield strength due to a decrease in the grain size, film thickness and high strain gradients, in nanocontacts specific features associated with the nature of the energy dissipation and friction in the nano- and atomic scale may occur.

To correctly determinate all the above mentioned values it is necessary that the depth of indentation at the local loading would not exceeded $\sim 1 / 10$ of the film thickness $\delta$, which requires the controlled application of forces to the probe in the micro- or mille-newton range. Modern equipment allows testing the films with a thickness from a few to tens of nanometers, and more.

As an example, let us consider the data about NI method attestation of thin films and superlattices for tribological purposes (Fig. 15) [136], testifying to nonmonotonic dependence $H(\delta)$ and the possibility of obtaining in superlattices hardness greater than that of each separate layer.

The limiting case of a thin film is a single-atomic layer, in particular, a monolayer of carbon atoms graphene, which was obtained several years ago [138-140]. The atoms in graphene have strong bonds arising from $s p_{2}$-hybridization of valence electrons [141-144]. So far the experimental data on the strength of these structures is quite insufficient [145]. One can only expect that they will be close to the corresponding mechanical properties of single-walled nanotubes, with which they have much in common.

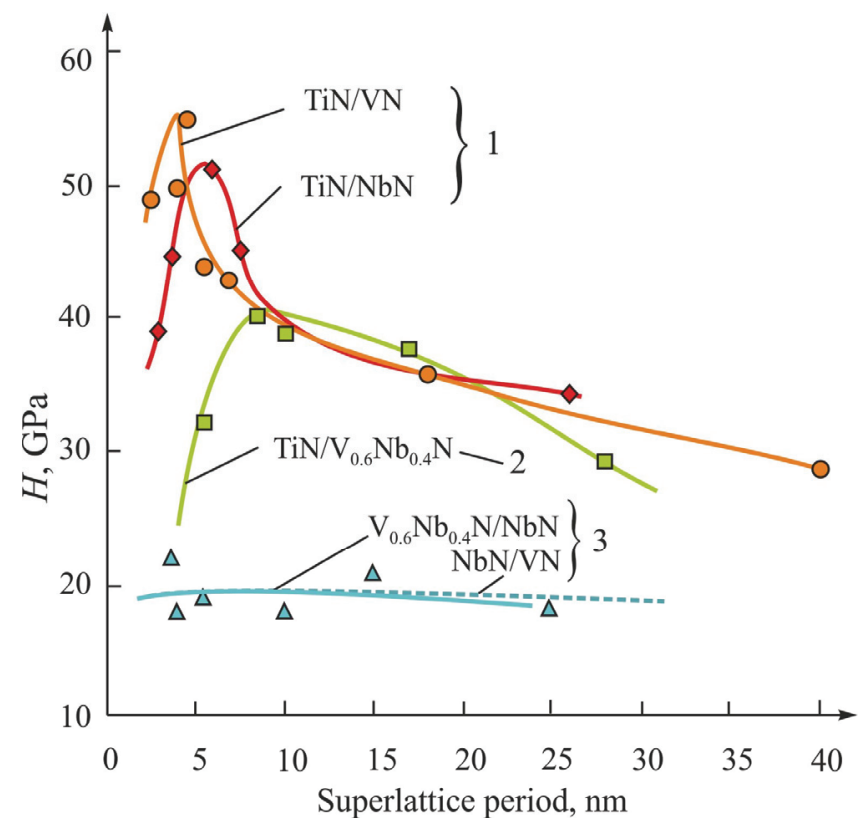

Fig. 15. The hardness of multilayer coatings of alternate layers of some nitrides as a function of the superlattice period:

$1-\Delta E \neq 0, \Delta a_{0} \neq 0 ; 2-\Delta E \neq 0, \Delta a_{0}=0 ; 3-\Delta E=0, \Delta a_{0} \neq 0$

( $a_{0}$ - superlattice parameter, $\Delta E$ and $\Delta a_{0}$ means the difference

in Young's modulus and parameters of the lattice of contacting layers, respectively) [136]

\section{Quasi-one-dimensional objects}

Since the middle of the last century, when the first filamentary crystals ("whiskers") with micrometer cross-sectional dimensions were synthesized, the studies of their mechanical properties have started. The miniaturization of MEMS/NEMS, sensor, nanomedicine and other equipment keeps going, therefore characterization of the mechanical properties of a substance in much smaller amounts, up to molecular ones, is becoming urgent.

\section{Nanopillars FIB-cut out from bulk material}

The authors of $[146,147]$ describe a technique for creating and testing samples with a cross-section in the submicro- and nanoscale. It implies the formation of a pillar sample in the material mass by a focused ion beam (FIB) in the column of a dual-beam scanning electron microscope (dual beam SEM). Then the formed pillar is deformed by the load (Fig. 16) produced by the head of the nanoindenter which has a flat tip.

Some of the results obtained in this way are shown in Fig. 17 and 18 [148-150]. From the diagrams of loading and the appearance of deformed Ni samples it is seen that a decrease in their diameter results in a replacement of a smooth mode of plastic flow by the hopping one after reaching critical stresses $\sigma_{\mathrm{c}}$. The value $\sigma_{\mathrm{c}}$ increased with decreasing $d$ according to the power law with exponent $n=-0.64$.

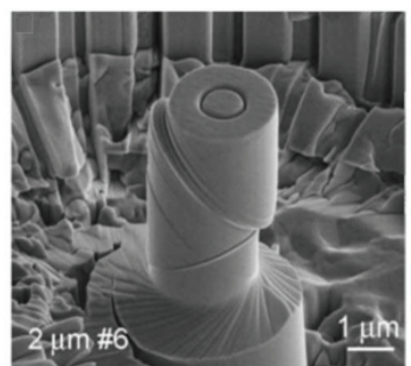

a)

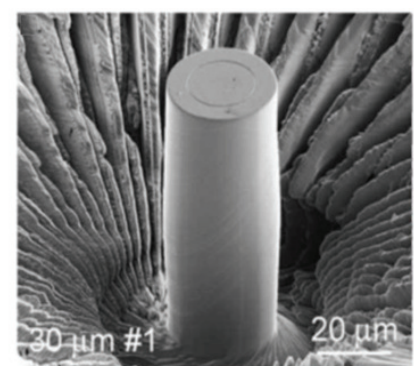

b)
Fig. 16. The appearance of single-crystal Ni sample formed by FIB and then deformed by uniaxial compression: $a-d=2 \mu \mathrm{m} ; b-d=30 \mu \mathrm{m}$

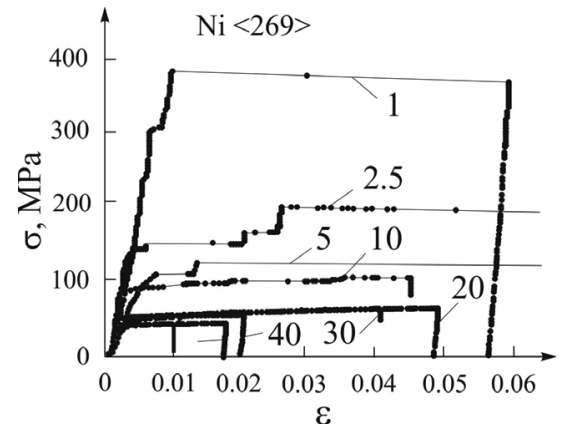

Fig. 17. The curves of deformation in $\sigma-\varepsilon$ coordinates for pure single crystal $\mathrm{Ni}$ in orientation $<269>$ (near the curves diameter $d$ of the sample is indicated in micrometers) 


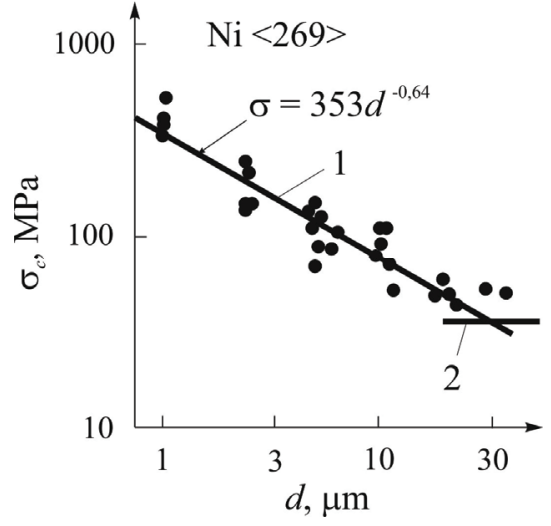

Fig. 18. The dependence of critical stresses of the compression on the diameter $d$ of Ni sample: 1 - microsample $d<30$ micrometers; 2 - bulk samples

Similar data were obtained by this method with other materials having a cubic lattice: $\mathrm{Au}, \mathrm{Al}, \mathrm{Cu}, \mathrm{LiF}$ [151-163] (Fig. 19). Value $n$ for them ranged from -0.6 to -0.81 . It was significantly higher than the exponent in the Hall-Petch law $(n=-0.5)$. In addition, the strengthening according to Hall-Petch implies the existence of internal borders in the material (between the grains, phases) that inhibit the motion of dislocations and increase their density in the crystal. In the case under discussion the boundaries (the surface of the sample) are free and are rather the sources and sinks for dislocations than barriers.

The authors of [148] offer the following explanation of the observed SE. In the macroscopic sample, there are many sources for dislocations: a double cross slip, Frank-Read sources, single-pole sources, grain boundaries, stress concentrators on the surface, and others. Therefore, with few exceptions, plastic flow stress is determined by the mechanism of inhibition of mobile dislocations: Peierls relief, point defects and their complexes, immobile "forest dislocations" being in intersecting planes, internal interfaces, and others. In the samples of sub-micron and nano-cross-section due to the rapid emergence of dislocations on the free surface, the limiting plasticity factor may not be the dislocation mobility but the speed of their generation $\dot{\rho}$ (especially in the ballistic regime, i.e. when the mean free path $\lambda>d$ ). Then $\dot{\varepsilon}=\dot{\rho} b \lambda$. For $\dot{\rho}=\partial \rho_{m} / \partial t$ we can write:

$$
\frac{\partial \rho_{m}}{\partial t}=\left(\delta_{h} \rho_{t}-\delta_{e} \frac{\lambda}{d} \rho_{m}-\delta_{t} \rho_{m}\right)<v>,
$$

where $\delta_{h}, \delta_{e}$ and $\delta_{t}$ are coefficients of dislocation multiplication, their obtaining as a result of runoff on the surface of the sample and their capture by stoppers (e.g., forest dislocations), $\rho_{t}$ и $\rho_{m}$ are total dislocation

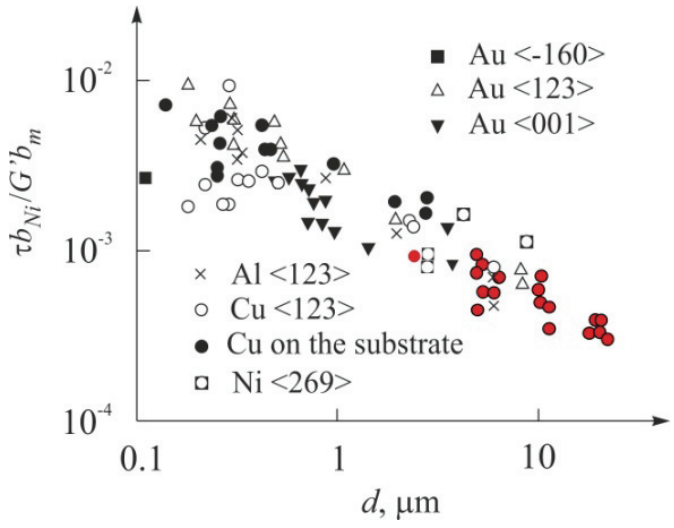

Fig. 19. The dependence of normalized critical stresses on the sample diameter in some FCC-metals [151]

density and density of mobile dislocations, respectively, $\langle v\rangle$ is average dislocation movement velocity.

$\mathrm{SE}$ analysis for critical flow stress $\sigma_{\mathrm{c}}$, carried out for this case in [148-150] shows that for certain relations $\delta_{h}, \delta_{e}$ and $\delta_{t}$ value $\sigma_{\mathrm{c}}$ varies in proportion to $d^{-3 / 4}$, which is consistent with experimental data. Thus, under deformation of the pillars with nano-/ submicroscale cross-section, the reasons for SE may occur which differ from those in polycrystalline bulk samples that provides an even stronger dependence $\sigma_{c}$ on $d$ than according to Hall-Petch.

Similar behavior data on nanopillars fabricated from amorphous alloys [152-163] testify to the SE absence in them, at least when $d$ is reduced to about $\sim 10 \mathrm{~nm}$ (Fig. 20).

Paper [164] contains some interesting data on the size effect in the alloy having shape memory $\mathrm{Cu}_{81,3} \mathrm{Al}_{13,7} \mathrm{Ni}_{5}$ (wt \%). It had small temperature hysteresis in the transformation of the cubic austenitic phase $\mathrm{L}_{2}$ into monoclinic martensitic $\beta^{\prime}$ and back.

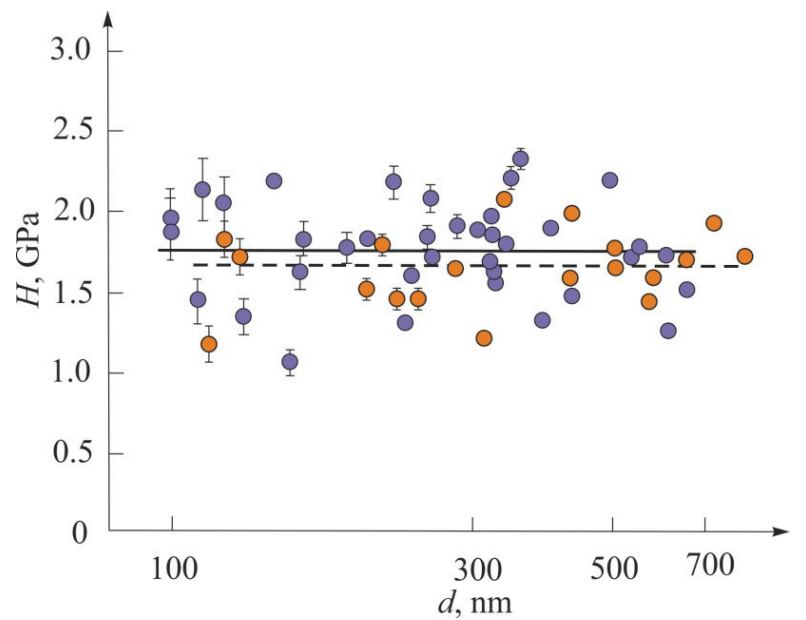

Fig. 20. The dependence of the yield strength on the nanopillar diameter for two amorphous alloys [152] 


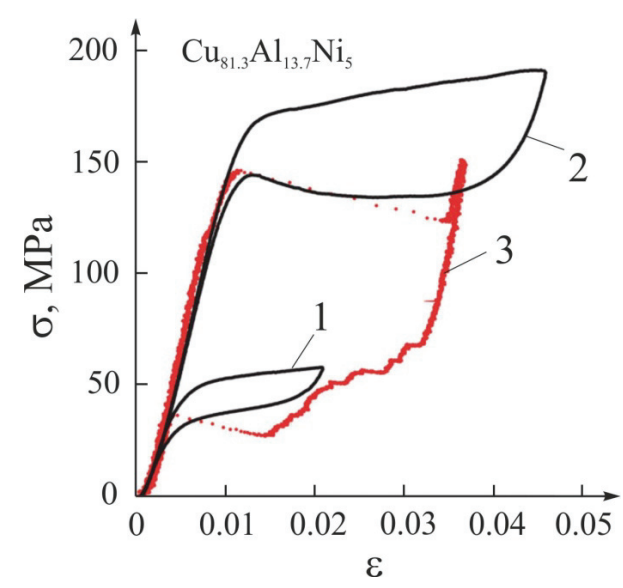

Fig. 21. The mechanical hysteresis loops in the shape memory alloy $\mathrm{Cu}_{81.3} \mathrm{Al}_{13.7} \mathrm{Ni}_{\mathbf{5}}$ :

1 and 2 - in a macro-sample at a temperature of 305 and $363 \mathrm{~K}$, respectively; 3 - in a column with a diameter of $750 \mathrm{~nm}$, a height of 3.8 microns at $T=295 \mathrm{~K}$. The rate of loading and unloading is $250 \mathrm{mN} / \mathrm{s}$ [164]

The corresponding temperatures for martensitic phase were $M_{s}=291 \mathrm{~K}, M_{f}=273 \mathrm{~K}$, and austenitic $A_{s}=285 \mathrm{~K}, A_{f}=303 \mathrm{~K}$ (here the indices $s$ and $f$ mean the beginning and end of the process, respectively). By means of FIB in the column of a scanning electron microscope the pillars with a diameter of $750 \mathrm{~nm}$ and a height of 3.8 microns were cut out of bulk material. Compression testing was performed using a Hysitron Triboindenter-750 with sphero-conical tip having rounding at the top with a radius of $600 \mathrm{~nm}$. Reference bulk samples were deformed by traditional methods.

The main result is shown in Fig. 21, from which it follows that the mechanical hysteresis loop area for submicro-sample increased several times compared with a bulk sample. Comparison of damping capacities of the nanopillars from alloy $\mathrm{Cu}-\mathrm{Al}-\mathrm{Ni}$ with other materials used to absorb vibrations, according to the criterion of damping quality $M=E^{1 / 2} \eta_{d}$ (here $\eta_{d}=\Delta W / \pi W_{\max }$ is loss factor, $\Delta W$ is absorbed energy, $W_{\max }$ is maximum energy stored in the cycle) shows that nanopillars have damping capacities several times more than macrosamples of the same alloy, as well as all known materials used for this purpose. Such properties of nanorods create good prospects for their use in microdevices, sensors, NEMS, etc. for damping parasitic oscillations and vibrations.

\section{Nanofibers}

Fibers of submicron and nanoscale cross-sections may be produced in various ways. This section will focus on electro-spinning - one of the simplest and universal techniques, at least for obtaining a wide range of polymeric and composite materials with a polymer matrix. The technique involves stretching a stream of molten or dissolved polymer injected from a fine nozzle towards a collector by electrostatic field of $\sim 1 \mathrm{kV} / \mathrm{cm}$ [165-168]. The electric field, overcoming surface tension, greatly reduces the diameter of a stream [169-181] (sometimes dividing it into several parallel ones) and can bring it to tens of nanometers. Stretching a fiber in liquid state and reducing its cross-section, by itself, leads to its hardening, but further effect can be achieved by adding reinforcing elements, in particular, carbon nanotubes (CNTs) to a solution (melt) [171, 176, 180].

Polymer nanofibers can be used at least in three ways:

a) as reinforcing elements for strengthening bulk nanocomposites or threads twisted of these fibers;

b) in sensing elements of sensor systems, electrochemical cell electrodes;

c) being folded in a random way into a nonwoven mat - like openwork matrices/ membranes with a low hydrodynamic resistance to contain catalysts, sorbents, pharmaceuticals, filter materials, etc.

In the process of producing nanofibres by electrospinning, nanoparticles, in particular, carbon nanotubes can be added to raw materials [171-180], that significantly increases the Young's modulus and strength (Fig. 22) [176], adds conductivity [170, 174, 177] increases the coefficient of absorption of microwave radiation [182], etc. A large specific surface area, ease of functionalization (both during production and thereafter), high strength, adjustable electrical conductivity and other characteristics of polymer nanofibers, reinforced with CNTs offer broad prospects for their use in biomedicine (for tissue engineering, enzyme immobilization, targeted drug delivery, filtering and decontamination of various media $[169,171])$.

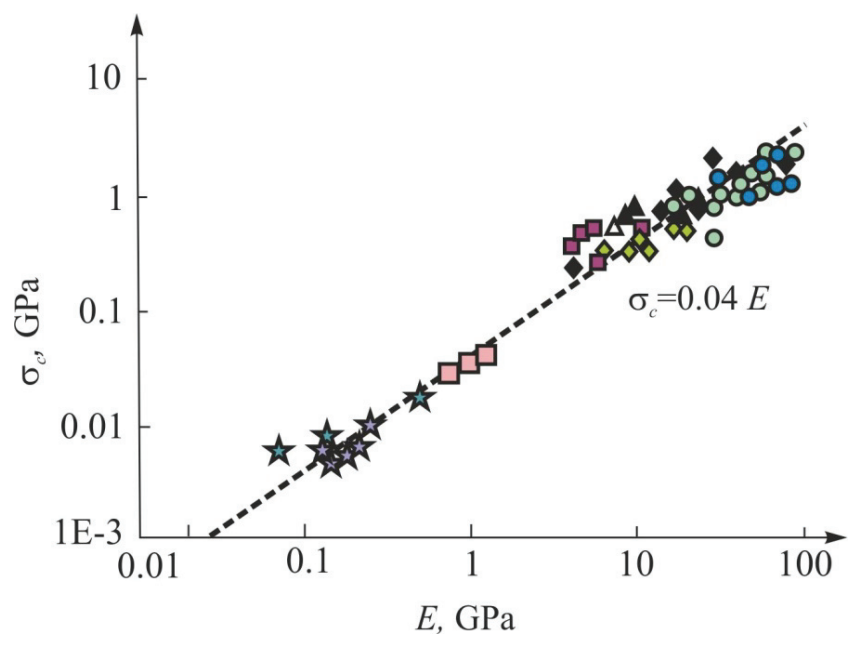

Fig. 22. The dependence of the strength of the polymer nanofiber reinforced with carbon nanotubes on the Young's modulus [176]

(Various signs indicate data of different authors) 


\section{Nanotubes}

In recent years, there have been synthesized a number of nanorod-containing structures, mechanical properties of which are of great interest. Experimental data available on the topic are not numerous and, being obtained by various methods, they do not agree. There are obvious difficulties of direct measurement of such characteristics as elastic modules, yield strength and ultimate strength, the conditions of elastic stability loss of individual nanoscale objects. On the other hand, theoretical and computational methods include the assumptions that cause some uncertainty in the results. For example, to calculate all the above characteristics (per se, macroscopic), the applied force should be normalized by the cross-section of thenanotube/fiber. When fibers, rods, or tubes have atomic sizes, their diameter and wall thickness can be set with an accuracy of tenths of effective atomic diameter, which causes the uncertainty. The smaller is the cross-section of the object, the greater is the uncertainty. Then the question arises about the appropriateness and correctness of the characterization of the mechanical properties of the objects of molecular size by means of concepts and values used in the theory of elasticity for continuous media.

Let us first consider a single-walled carbon tube (SWCNT) as the most extensively studied quasi-onedimensional tubular nanostructure. It can be presented as rolled up graphene sheet in the form of a cylindrical shell. The bond deformation energy of a graphene layer of such curved configuration is weakly dependent on the chirality of the tube, but depends quite substantially on the tube radius $R_{t}$.

However, at $R_{t} \gtrsim 0.7 \mathrm{~nm}$, this energy is not great $(\sim 0.05 \mathrm{eV} /$ atom $)$ and slightly decreases with increasing $R_{t}$. Therefore, the elastic moduli (for example, Young's) should be close to previously found longitudinal compression moduli of graphene sheets $(E=1.06 \mathrm{TPa})$. The calculations and experiments done provide SWSNT with values $E$ from 0.8 to $1.28 \mathrm{TPa}$ with an error as high as $30 \%$ [18, $183-186]$. Note that value $E \sim 1 \mathrm{TPa}$ is inherent only in defect-free single-walled nanotubes grown by arc or laser ablation (PVD) techniques. The tubes synthesized by more productive CVD processes of catalytic pyrolysis of hydrocarbons have a multilayered structure (MWCNT) and contain much more structural defects than SWCNTs. Therefore, the Young's modulus of real multilayered tubes is usually one or often two orders of magnitude less than that of singlelayered CNTs [187-198].
In addition to pure carbon nanotubes, other tubular structures of simple chemical compounds $\left(\mathrm{TiO}_{2}, \mathrm{ZrO}_{2}, \mathrm{CuS}, \mathrm{BN}, \mathrm{BC}_{3}, \mathrm{BC}_{2} \mathrm{~N}, \mathrm{C}_{3} \mathrm{~N}_{4}, \mathrm{CN}, \mathrm{WS}_{2}\right.$, $\mathrm{MoS}_{2}$, etc.) are synthesized but in much smaller quantities [199-203]. All of them have lower elastic moduli than those of carbon nanotubes. Nanoropes consisting of twisted bundles of nanotubes have lower values of elastic moduli (0.5-0.8 TPa), apparently due to ignoring the voids between individual tubes. Poisson's ratio calculated for carbon nanotubes is always positive, slightly depending on their chirality and ranges from 0.12 to 0.2 , and for $\mathrm{BN}$ nanotubes from 0.23 to 0.3 .

Of no less importance are yield strength, ultimate stresses, which can be withstood by a tube under stretching, compression, bending, etc. From general considerations made above, the yield stress and strength can not exceed one or two tenths of $E$ and, hence, for MWCNT they must amount to several GPa, and for SWCNT about a hundred GPa. Measuring these characteristics on single tubes appears to be even harder than the elastic moduli. Typically, to reduce the measurement error to an acceptable value a bundle of tubes or fibers is tested. A few experimental data show that tensile strength of high-quality single-walled nanotubes obtained by PVD method can reach $\sim 50 \mathrm{GPa}$, which is 20 times as much as that of highstrength structural steels (at a density 6 times less). As expected, CVD-synthesized tubes have the strength ten times less.

Like all covalent carbon materials, nanotubes are destroyed at a low homologous temperature. In [204] the technique of plasticization of nanotubes by passing an electric current in the process of deformation was proposed. For stretching nanotubes of various diameters comprising a different number of layers, a piezoceramic manipulator was used. The initial length of all the tubes was $24 \mathrm{~nm}$. The current density during the test was $10 \mathrm{MA} / \mathrm{mm}^{2}$ ! Under these conditions the samples were destroyed at a large residual strain (over $300 \%$ !). Even in metals capability for such large deformations is called superplasticity. Large electroplastic deformation was observed in all tested nanotubes (single, double and multi-walled). Thus, a tube of $12 \mathrm{~nm}$ in initial diameter after the test had a diameter of $0.8 \mathrm{~nm}$ and a length of $91 \mathrm{~nm}$ which is 3.79 times greater than the original.

Mechanical deformation can change electronic properties of nanotubes, which manifests itself in variations of transport characteristics, and charge imposition leads to a significant deformation. Such electro-mechanical effects can be used in sensors, manipulators, memory cells, etc. 
In addition to applications in nanoelectronics, sensing, scanning probe microscopy, and other areas of nanotech, nanotubes are beginning to be used for reinforcement of composite functional and structural materials with metallic, ceramic and polymer matrix [205-213]. In the latter case, long-used carbon fibers with micrometer cross-section are replaced by nanotubes. For this purpose, relatively inexpensive multi-walled tubes obtained by CVD methods are used. They have low strength characteristics, so significant reinforcement of composites has not been achieved yet (maximum - a few times).

It was found that at low concentrations of tubes in the matrix strengthening effect is not due to the additive contribution of nanotubes (in the described cases, it is usually negligible), but rather to the changes in the conditions of nucleation and growth of the matrix crystallites (if it is created in the presence of nanotubes from solution, electrolyte, etc.). Due to the grinding of grains, boundary structure changes, porosity decrease, and other factors hardening of composite can be achieved by tens of percent at volume concentration of tubes only $10^{-2}-10^{-1} \%$.

\section{Macromolecules}

The limiting case of nanomechanical tests of onedimensional nano-objects is mechanical spectroscopy of single macromolecules and paired interactions between them. It has a number of advantages over the methods of research of large ensembles of molecules. Since the initiation of the reaction in the macro- or the microreactor (even if it occurs simultaneously in the entire volume, which is the idealization of actual conditions) the states of single molecules in the ensemble quickly desynchronize due to accidental causes (thermal fluctuations, collisions, heterogeneity of physical fields, local composition and so on). As a result, the traditional study of chemical kinetics in macro-volumes deals with ensembles of desynchronized molecules and provides information on the average ensemble states.

This does not allow controlling the intermediates, the intermediate states and products. Studies at the level of single molecules do not require synchronization and in principle allow one to detect and characterize the intermediate conformations and states of macromolecules, i.e. identify and detail the atomic mechanisms of chemical reactions in real-time by direct measurements, rather than indirectly. Application of the controlled force to a single molecule provides a new tool (alongside with temperature, pressure, light, etc.) to study the thermodynamics and kinetics of reactions involving macromolecules. In particular, one can determine which molecular domains and which molecule lengths interact in DNA and RNA polymerase, what are the characteristic times of limiting steps, the structure of active centres in enzymes, etc.

In recent years these methods have been used to study the mechanisms of chemical and catalytic reactions, in which the conformation of macromolecules substantially changes in the process of their occurrence. There are a number of reviews and monographs on this topic $[68,69,72]$. For example, the book [68] is entirely devoted to the physical foundations, approaches, techniques and devices for mechanical spectroscopy of single molecules and individual bonds between them. It contains reviews and comparative analysis of the well known techniques and results obtained with their help, followed by an extensive bibliography. The results presented deal with the study of protein molecules (including enzymes), peptides, saccharides, DNA, RNA, ribosomes, actin, myosin, kinesin, dynein, streptavidin, molecular
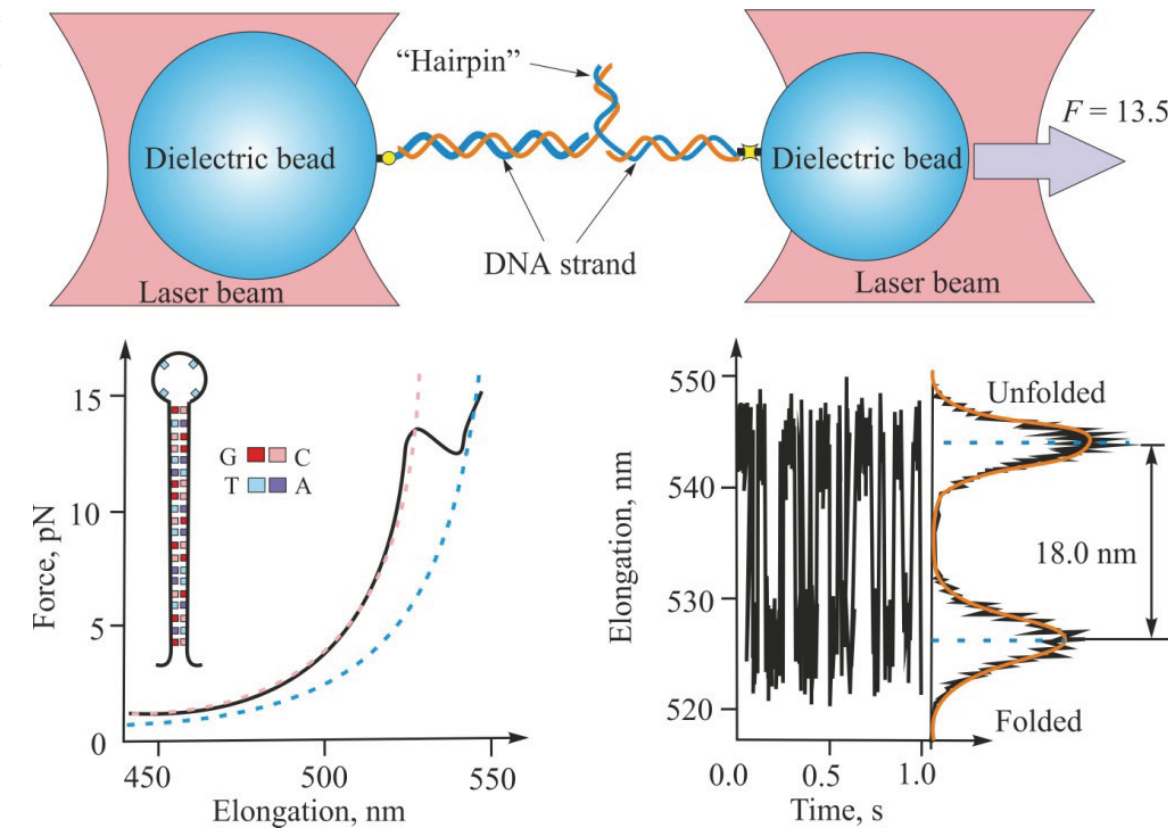

Fig. 23. The study of the dynamics of unfolding/folding "hairpins" of the DNA molecule by double optical tweezers method [217]: $a$-experimental scheme, $b$-dependence of the applied force on elongation, $c$ - dependence of elongation on time at a constant applied force $F=13.5 \mathrm{pN}$ 
complexes, membranes, and cytoplasm of living cells. Here are some typical examples of the results.

In [214] a number of specific methods and results of AFM application are presented. They are aimed at determining the bonding force and energy in molecular complexes (in particular, protein-protein, proteinligand). It is shown that by varying the speed of application of tensile force one can estimate the affinity, rate constants of individual bonds rupture, some of the structural features of the bond points and recognition mechanisms.

In [215] the technique of the combined atomicforce and fluorescence microscopy is described. It provides $\sim 1 \mathrm{~nm}$ resolution monitoring of the changes in the conformation of a single protein molecule when it is stretched and under the action of the applied force of about $100 \mathrm{pN}$. The [216] describes high-resolution (both in space and in time) methods for observing molecular movements (particularly, fluorescent resonant energy transfer, etc.).

The authors of [217] studied the effect of the stretching force generated by optical tweezers on thermodynamics and kinetics of reactions involving single DNA molecules (Fig. 23). It is found that application of the force in the range $0-25 \mathrm{pN}$ leads to a change in the conformation of the molecule, triggers or blocks some reactions, changes activation energy which can accelerate or decelerate the reaction.

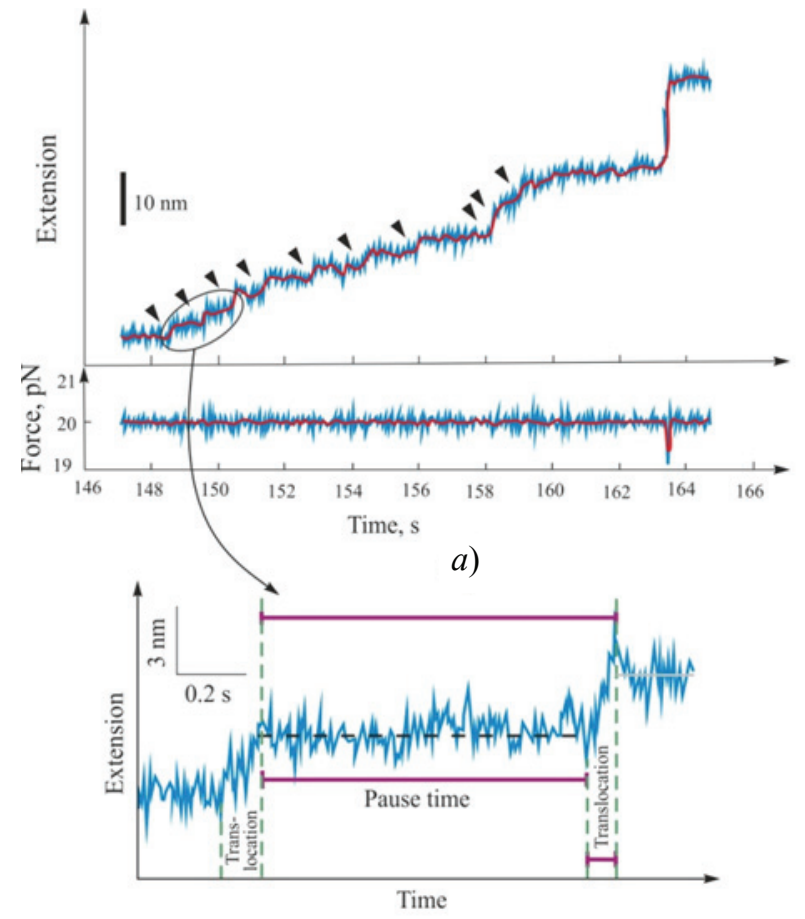

b)

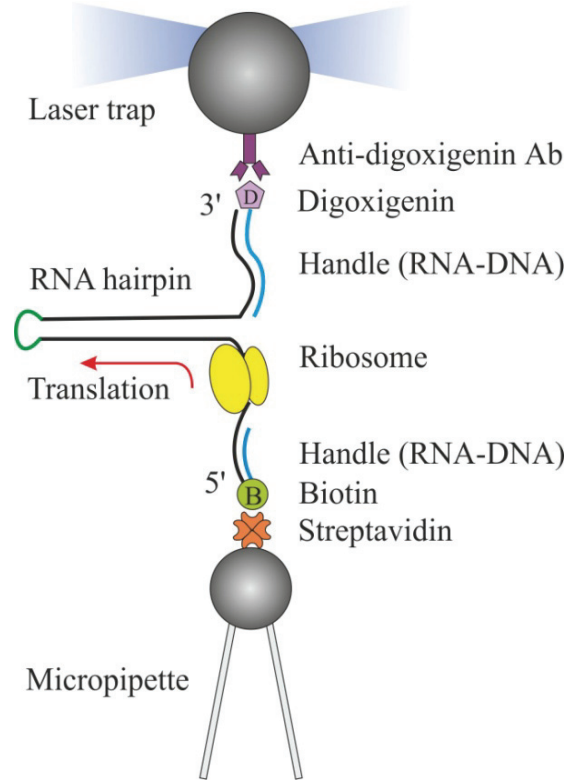

Fig. 24. The scheme of the experiment to determine the mechanism of codon translation [218]

The pioneering work [218] presents the results of the real-time study (by application of constant force of $10-20 \mathrm{pN}$ to the "hairpin" of RNA molecule) of the dynamics and mechanisms of one codon translation by a single ribosome (Fig. 24). Single stages, their duration and the effect of force applied to them have been identified (Fig. 25).
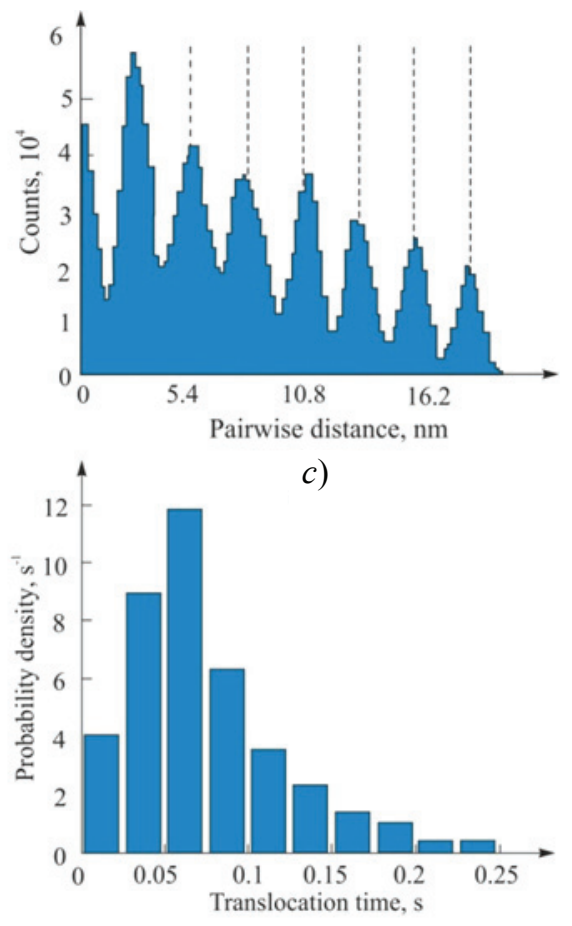

d)

Fig. 25. Dynamics of real time-translation of codon [218]:

$a$ - the dependence of elongation and applied force on time; $b$ - the dependence of elongation on time for elementary deformation jump; $c$ - length distribution of deformation jumps; $d$ - latency distribution of deformation jumps 
Methods of force spectroscopy of single molecules in [219] were used to study the mechanism of catalytic reduction of the disulfide bonds by the thioredoxin Escherica coli enzyme. It is well known that this process is vital for all living organisms. Two alternative forms of catalytic reactions have been found: the first is implemented through a reorientation of the disulfide bond of substrate polypeptide and causes its shortening by $(0.79 \pm 0.09) \mathrm{nm}$, and the second - elongation by $(0.17 \pm 0.02) \mathrm{nm}$. This supports the idea that catalytic activity of thioredoxin is due to prior precise repositioning of reacting sulfur atoms. The results described can be important for understanding the mechanisms regulating the activity of thioredoxin under the oxidative stress and mechanical damage that arises in the cardiovascular diseases.

Along with bipolar optical and magnetic tweezers, multipolar tweezers $(3,4$ or more poles) are often used to enhance spatial manipulation. Thus, in the [220] using magnetic microbeads $\left(47 \% \gamma-\mathrm{Fe}_{3} \mathrm{O}_{4}\right)$ of 0.35 and $1.05 \mu \mathrm{m}$ in diameter and three-pole magnetic tweezers (gradient magnetic field $\sim 8 \cdot 10^{3} \mathrm{~T} / \mathrm{m}$ ), the rheological properties of living cells cytoplasm (by the speed of magnetic probe displacement inside the cell) were studied. This makes it possible to set the state and nature of cytoskeleton molecule cross-linking.

\section{Conclusion}

Low-dimensional and nanostructured materials are characterized by a number of features of the mechanical behaviour that lead to significant and often drastic changes in their physical and mechanical properties as compared with bulk materials having the same chemical composition. These features, and, as a rule, much higher strength of low-dimensional nanostructured materials enable the creation of products with unique characteristics unattainable with conventional materials. However, insufficient knowledge of nanomaterials and incorrect use of reference data related to the bulk materials in the construction of nanomaterials require additional studies. They need to reveal the regularities of occurrence of size effects in mechanical properties and their physical nature at the atomic and molecular structure level.

\section{Acknowledgment}

This work was supported by the Russian Science Foundation (RSF) grant15-19-00181 (Techniques and analysis of nanomechanical tests, causes of size effects on yield and strength of solids); RSF grant 14-13-00731 (Mechanical behaviour of single macromolecules); the grant of the Ministry of Education and Science of the Russian Federation K1-2014-022 (Ways and means of controlling the mechanical behaviour of biological macromolecules).

\section{References}

99. Glezer, A.M. and Permyakova, I.E. (2013). MeltQuenched Nanocrystals. CRC Press, 369 p.

100. Suryanarayana, C. and Inoue, A. (2011). Bulk Metallic Glasses. Boca Raton, Fl.: CRC Press. 523 p.

101. Greer, A.L. (2009). Materials Today, 12, 14.

102. Miller, M. and Liaw, P., ed. (2008). Bulk Metallic Glasses. New York: Springer Science, $237 \mathrm{p}$.

103. Sahu, K.K., et al. (2010) Acta Mater., 58, 4199.

104. Cheng, Y.Q. and Ma, E. (2011). Progress in Mater. Sci., 56, 379. 4804.

105. Ohodnicki, P.R., et al. (2010). Acta Mater., 58,

106. Wang, D.J., et al. (2010). Scripta Mater., 63, 764.

107. Wu, Y., et al. (2011) Acta Mater., 59, 2928.

108. Maas, R., Klaumünzez, D. and Löffler, J.F. (2011). Acta Mater., 59, 3205.

109. Chen, W., et al. (2011). Mater. Letters, 65. 1172.

110. Concustell, A., et al. (2011). Scripta Mater., 64, 1091. 2221

111. Qin, W., Szpunar, J.A. and Umakoshi, Y. (2011). Acta Mater., 59, 2221.

112. Bokeloh, N., et al. (2010). Acta Mater., 58, 3919.

113. Trexler, M.M. and Thadhani, N.N. (2011). Progress in Mater Sci., 55, 759.

114. Schroers, J., et al. (20 11). Mater Today, 14, 14.

115. Pogrebnyak, A.D., et al. (2009). Uspehi fizicheskih nauk [Advances in physical sciences], 179, 35.

116. Fornell, J., et al. (2010). Acta Mater., 58, 6256.

117. Chen, N., et al. (2010). Acta Mater., 58, 5886.

118. Wang, D.J., et al. (2010. Scripta Mater., 63, 764.

119. Wu. Y., et al. (2011). Acta Mater., 59, 2928.

120. Miracle, D.B., et al. (2011). Acta Mater., 59, 2831.

121. Lee, C.J., et al. (2010). Scripta Mater., 63, 105.

122. Gleiter, H. (2000). Acta Mater., 48, 1.

123. Koch ,C.C., et al. (2010). J. Mater. Sci., 45, 4725.

124. Massart, T.J. and Pardoen, T. (2010). Acta Mater., 58, 5768.

125. Wang, Q.G., et al. (2010). Acta Mater., 58, 3006.

126. Yue, L., Zhang, H. and Li, D.Y. (2010). Acta Mater., 58, 2677.

127. Guisbiers, G. (2010). Nanoscale res. Lett., 5, 1132.

128. Ahn, R., et al. (2010). J. Mater. Sci., 45, 4790.

129. Dehm, G. (2010). Progress in Molecular Science, 54,664

130. Barry, C.C. and Grant, N.M. (2007). Ceramic Materials. Science and Engineering. Berlin, Heidelberg: Springer, $716 \mathrm{p}$. 
131. Shakelford, J.F. and Doremus, R.H., ed. (2008). Ceramic and Glass Materials. Structure, Properties and Processing. New York: Springer Science, 201 p.

132. Koch, C.C., et al. (2007). Structural Nanocrystalline Materials. Fundamental and Applications. Cambridge, UK: Cambridge University Press, 364 p.

133. Yue, L., Zhang, H. and Li, D.Y. (2010). Acta Mater., 58, 2677.

134. Fan, G.J., et al. (2006). Acta Mater., 54, 1759.

135. Ovid'ko, I.A. and Sheinerman, A.G. (2007). Rev. Adv. Mater. Sci., 16, 1.

136. Cavaliero, A. and De Hosson, J.Th.M., ed. (2006). Nanostructured Coatings. New York: Springer, $648 \mathrm{p}$.

137. Zhang, J.Y., et al. (2010). Scripta Mater., 63, 101.

138. Novoselov, K.S., et al. (2004). Science, 306, 666.

139. Geim, A.K. (2008). Science, 324, 1530.

140. Geim, A.K. and Novoselov, K.S. (2007). Nature Materials., 6, 183.

141. Terrones, M., et al. (2010). Nano Today, 5, 351.

142. Kim, J., Kim, F. and Huang, J. (2010). Materials Today, 13, 28

143. Wassei, J.K. and Kaner, R.B. (2010). Materials Today, 13, 52.

144. Katsnelson, M.I. (2007). Materials Today, 10, 20.

145. Lee, C., et al. (2008). Science, 321, 385.

146. Dimiduk, D.M., Uchic M.D. and Parthasarathy,

T.A. (2005). Acta Mater., 53, 4065.

147. Uchic M.D., et al. (2004). Science, 305, 986.

148. Nadgorny, E.M., Dimiduk, D.M. and Uchic, M.

(2008). J. Mater. Res., 23, 2829.

149. Juan, J.M.S., No, M.L. and Schuh, C.A. (2008). Adv. Mater. 20, 272.

150. Dimiduk, D.M., et al. (2006). Science, 312, 1188.

151. Brown, J.A. and Ghonien, N.M. (2010). Acta Mater., 58, 886

152. Chen, C.Q., Pei, Y.T. and De Hosson, J.T.M. (2010). Acta Mater., 58, 189. 5789. 5160.

153. Brahathula, A., et al. (2010). Acta Mater., 58,

154. Lowry, M.B., et al. (2010). Acta Mater., 58,

155. Ng, K.S. and Ngan, A.H.W. (2009). Acta Mater., 57,4902

156. Yang Y., et al. (2009). Acta Mater., 57, 1613.

157. Richter, G. (2010). Scripta Mater., 63, 933.

158. Dou, R.B., Xu, B. and Derby, B. (2010). Scripta Mater., 63, 308. 62,746

159. Zimmermann, J., et al. (2010). Scripta Mater., 63,480

160. Jiang, I. and Chawla, N. (2010). Scripta Mater.,

161. Kiener, D., et al. (2010). Scripta Mater., 63, 500.

162. Jie, J., et al. (2010). Nano Today, 5, 313.

163. Li, Y., Zhang J. and Yang, B. (2010). Nano Today, 5, 117.

164. Juan, J.S., No, M.L. and Schuh, C.A. (2009). Nature nanotechnology, 4, 415.

165. Lukas, D., et al. (2009). Physical principles of electrospinning. Boca Raton, Fl.: Taylor \& Francis.
166. Haghi, A.K. and Zaikov, G.E. (2011). Electrospinning of Nanofibers: From Introduction to Application. New York: Nova Science Publishers, $186 \mathrm{p}$.

167. Haghi, A.K. and Zaikov, G.E. (2011). Nanofiber Research: Advances in Theory and Practice. New York: Nova Science Publishers, $186 \mathrm{p}$.

168. Hun, T. (2009). Electrospinning and Nanofibres. VDM Velarg, $120 \mathrm{p}$.

169. Rutledge, G.C. and Fridrikh, S.V. (2007). Advanced Drag Delivery Review, 59, 1384.

170. Agarwal, S., Wendorff, J.H. and Greiner, A. (2008). Polymer, 49, 5603.

171. Chen, H., Liu, Zh. and Cebe, P. (2009). Polymer, 50,872 .

172. Kim, M.J., et al. (2010). Syntetic Metals, 160, 1410.

173. Ding, B., et al. (2010). Materials Today, 13, 16.

174. Sundaray, B., Choi, A. and Park, Y.W. (2010). Synthetic Metals, 160, 984.

175. Reich, S. (2010). Composites Science and Technology, 70, 1584.

176. Almecija, D., et al. (2009). Carbon, 47, 2253.

177. Mazinani, S., Ajj, A. and Dubois, C. (2009). Polymer, 50, 3329.

178. Gandhi, M., et al. (2009). Polymer, 50, 1918.

179. Kang, M., Chen, P. and Jin, H.-J. (2009). Current Applied Physics, 9, 95.

180. Wu, D., et al. (2011). European Polymer Journal, 47, 284.

181. Manesh, K.M., et al. (2008). Biosensors and Bioelectronics. 23, 771 .

182. Mihaylin, Yu.A. (2011). Spetsialnyie polimernyie kompozitsionnyie materialyi [Special polymer composite materials]. S.-P.: Nauchnyie osnovyi i tehnologii, 660 p.

183. Harris, P.J.F. (2009). Carbon Nanotube Science. Cambridge: Cambridge University Press, $301 \mathrm{p}$.

184. Ganesan, Y. and Lou, J. (2010). JOM, 61, 32.

185. Jorio, A., Dresselhaus, G. and Dresselhaus, M.S., ed. (2008). Carbon Nanotubes. Berlin, Heidelberg: Springer-Velarg, $720 \mathrm{p}$.

186. Tjong, S.C. (2009). Carbon Nanotube Reinforced Composites. Weinheim.: Wiley-VCN, 228 p.

187. Kuemmeth, F., et al. (2010). Materials Today, 13,18 .

188. Ferralis, N. (2010). J. Mater. Sci., 45, 5135.

189. Balani, K., et al. (2009). JOM, 61, 63.

190. Zhang, H., et al. (2010). J. Mater. Sci., 45, 5759.

191. Kang, J. (2009). J. Mater. Sci., 44, 5602.

192. Cho, J., Boccaccini, A.R. and Shaffer M.S.P. (2009). J. Mater. Sci., 44, 1934.

193. Suhr, J. and Koratkaz, N. (2008). J. Mater. Sci., 43,4370

194. Kao, C. and Young, R.J. (2010). J. Mater. Sci., $45,1425$.

195. Zheng, C., et al. (2011). Materials Letters, 65, 150.

196. Mediana-Gonzalez, Y. and Remigy, J.-C. (2011). Materials Letters, 65, 229.

197. Castro, R.H.R., Hidalgo, P. and Dimiz, E.C. (20110. Materials Letters, 65, 271.

198. Bi, S., et al. (2010). Physica, B.405, 3312.

199. Li, H., et al. (2011). Materials Letters, 65, 1188. 
200. Bavykin, D.V. and Walsh, F.C. (2010). Titanate and Titania Nanotubes. Syntesis, Properties and Applications. Cambrige. UK: RSC Publishing, 154 p.

201. Liu, X.-L. and Zhu, Y.J. (2011). Materials Letters, 65, 1089.

202. Harrison, M.A., et al. (2011). Materials Letters, 65,420 .

203. Li, L., et al. (2011). Materials Letters, 65, 1434.

204. Huang, J.Y. (2006). Nature, 439, 281.

205. Zhang, F.-H., et al. (2009). J. Mater. Sci., 44, 3574.

206. Joo, S.-H., et al. (2010). J. Mater. Sci., 45, 4652.

207. Sridhar, I. and Narayanan, K.R. (2009). J. Mater. Sci., 44, 1750.

208. Salinas-Ruiz, M.M., Skordos, A.A. and Partridge, I.K. (2010). J. Mater. Sci., 45, 2633.

209. Rakhimkulov, A.D., et al. (2010). J. Mater. Sci., 45,633 .
210. Hvizdos, P., et al. (2010). Scripta Mater., 63, 245.

211. Choi, H., et al. (2011). Scripta Mater., 64, 601.

212. Lahiri, D., et al. (2011). J. of the mechanical behavior of biomedical materials, 4, 44 .

213. Byrnet, E.M., et al. (2010). Acta Mater., 58, 6324.

214. Lee, C. K., et al. (2007). Micron, 38, 446.

215. Sarkar, A., Robertson, R.B. and Fernandez, J.M. (2004). PNAS, 101, 12882.

216. Greenleaf, W.J., Woodside, M. T. and Block, S.M. (2007). Annu. Rev. Biophys. Biol. Struct., 36, 171.

217. Tinoco, I. and Bustamante, C. (2002). Biophysical Chemistry, 101-102, 513.

218. Wen, J.-D. (2008). Nature, 452, 598.

219. Wiita, A.P., et al. (2007). Nature, 450, 124.

220. Vries de, A.H.B. (2005). Biophysical Journal, $88,2137$.

\section{International Scientific and Technical Conference Dedicated to the $105^{\text {th }}$ Anniversary of A. N. Planovsky (ISTC Planovsky - 2016)}

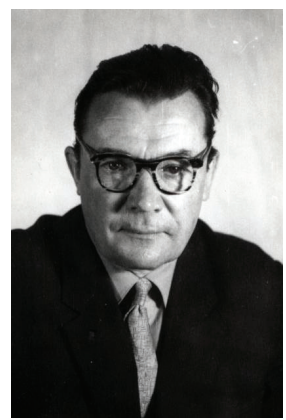

\section{International Scientific and Technical Conference \\ "Increasing the Effectiveness of Processes and Machines in Chemical and Related Industries" \\ Moscow \\ September 8-9, 2016}

Call for Proposals

- Hydro mechanical processes (separation of liquid and gas inhomogeneous systems, mixing of liquids, fluidization, pneumatic transport)

- Mass-transfer and heat-and-mass-transfer processes (absorption, rectification, liquid extraction, drying, adsorption, chemisorption, extraction from solid phase, membrane separation of mixtures)

- Chemical engineering processes in industry and agriculture

- Mechanical processes (grinding, classification, mixing, pressing, granulation)

- Processes and systems of technosphere protection (industrial and environmental safety of processes and machines)

- Management and optimization of technological processes. Methods and means of monitoring and management of technological processes.

The venue of conference plenary session:

Moscow State University of Design and Technology

\section{The Head of the Organizing Committee}

Professor Stanislav Rudobashta - Head of the Committee of the Russian Union of Scientific and Engineering Public Associations on Drying and Hydrothermal Treatment of Materials (phone number: 890559023 38, e-mail: rudobashta@mail.ru)

\section{Scientific Secretary of the Organizing Committee}

Professor Maria Kosheleva - Scientific Secretary of the Committee of the Russian Union of Scientific and Engineering Public Associations on Drying and Hydrothermal Treatment of Materials (phone number: 892635504 48, e-mail: oxtpaxt@ya.ru)

\section{Conference information support at the website} www.drying-committee.ru

Professor Vladimir Volynsky - Member the Bureau of Committee of the Russian Union of Scientific and Engineering Public Associations on Drying and Hydrothermal Treatment of Materials (phone number 891098505 60, 896124429 70, e-mail: vvolyn@mail.ru) 